\title{
Development and validation of a prognostic score predicting recurrence in resected combined hepatocellular cholangiocarcinoma
}

This article was published in the following Dove Press journal: Cancer Management and Research

\author{
Meng-Xin Tian, ${ }^{1,2, *}$ Liu- \\ Ping Luo, ${ }^{3,4, *}$ Wei-Ren Liu, ${ }^{1,2, *}$ \\ Wei Deng, ${ }^{5}$ Jia-Cheng Yin, ${ }^{1,2}$ \\ Lei Jin, ${ }^{1,2}$ Xi-Fei Jiang, ${ }^{1,2}$ Yu- \\ Fu Zhou, ${ }^{1,2}$ Wei-Feng Qu, ${ }^{1,2}$ \\ Zheng Tang, ${ }^{1,2}$ Han Wang, ${ }^{1,2}$ \\ Chen-Yang Tao, ${ }^{1,2}$ Yuan Fang, ${ }^{1,2}$ \\ Shuang-Jian Qiu, ${ }^{1,2}$ \\ Jian Zhou, ${ }^{1,2,6}$ Jing-Feng Liu, 3,4 \\ Jia Fan, ${ }^{1,2,6}$ Ying-Hong Shi, ${ }^{1,2}$ \\ 'Department of Liver Surgery, Liver Cancer \\ Institute, Zhongshan Hospital, Fudan University, \\ Shanghai, People's Republic of China; ${ }^{2}$ Key \\ Laboratory of Carcinogenesis and Cancer \\ Invasion of Ministry of Education, Shanghai, \\ People's Republic of China; ${ }^{3}$ Department of \\ Hepatobiliary Surgery, Mengchao Hepatobiliary \\ Hospital of Fujian Medical University, Fuzhou, \\ Fujian, People's Republic of China; ${ }^{4}$ The United \\ Innovation of Mengchao Hepatobiliary \\ Technology Key Laboratory of Fujian Province, \\ Mengchao Hepatobiliary Hospital of Fujian \\ Medical University, Fuzhou, Fujian, People's \\ Republic of China; ${ }^{5}$ Department of Health \\ Statistics and Social Medicine, Key Laboratory of \\ Public Health Safety, Ministry of Education, \\ School of Public Health, Fudan University, \\ Shanghai, People's Republic of China; ' Institutes \\ of Biomedical Sciences, Fudan University, \\ Shanghai, People's Republic of China \\ *These authors contributed equally to this work
}

Correspondence: Ying-Hong Shi Department of Liver Surgery, Liver Cancer Institute, Zhongshan Hospital, Fudan University, I80 FengLin Road, Shanghai 200032, People's Republic of China

Tel +8621 640419903233

Fax +86 21 6403 7181

Email shi.yinghong@zs-hospital.sh.cn

Jing-Feng Liu

The United Innovation of Mengchao Hepatobiliary Technology Key Laboratory of Fujian Province, Mengchao Hepatobiliary Hospital of Fujian Medical University, Xihong Road 312, Fuzhou 350025, Fujian, People's Republic of China, China

$\mathrm{Tel} / \mathrm{Fax}+8659183705927$

Email drjingfeng@।26.com
Purpose: To develop and validate a decision aid to help make individualized estimates of tumor recurrence for patients with resected combined hepatocellular cholangiocarcinoma (CHC).

Patients and methods: Risk factors of recurrence were identified in the derivation cohort of 208 patients who underwent liver resection between 1995 and 2014 at Zhongshan Hospital to develop a prediction score. The model was subsequently validated in an external cohort of 101 CHC patients using the C concordance statistic and net reclassification index (NRI).

Results: On multivariate analysis, five independent predictors associated with tumor recurrence were identified, including sex, $\gamma$-glutamyl transferase, macrovascular invasion, hilar lymphoid metastasis and adjuvant transcatheter arterial chemoembolization. The prediction score was constructed using these 5 variables, with scores ranging from 0 to 5 . A patient with a score of 0 had a predicted 1 - and 5-year recurrence risk of $11.1 \%$ and $22.2 \%$, respectively. In the validation cohort, the NRIs of prediction score vs American Joint Committee on Cancer $7^{\text {th }}$ TNM staging system at 1-year and 5-year were 0.185 (95\% CI, 0.090-0.279, $P<0.001)$ and 0.425 (95\% CI, 0.044-0.806, $P=0.03$ ), respectively.

Conclusion: Our developed and validated prediction score might be a simple and reliable method in postoperative CHC patients and help clinicians identify candidates who may benefit from future adjuvant therapies.

Keywords: combined hepatocellular cholangiocarcinoma, recurrence prediction, prognosis, liver resection

\section{Introduction}

Combined hepatocellular cholangiocarcinoma $(\mathrm{CHC})$ is a rare liver malignancy, accounting for $0.4 \sim 14.2 \%$ of all primary liver cancers. ${ }^{1}$ It is comprised of dual histologic features: hepatocellular and biliary epithelial differentiation. Since the first report of Allen and Lisa in $1949,{ }^{2}$ there have been an increasing number of clinical studies describing both the demographic and clinical features of $\mathrm{CHC}{ }^{3-5}$ Due to the limitations of small study populations in previous reports, the demographic, clinical characteristics, and prognostic factors are far from clearly understood. ${ }^{6}$

Over the past decades, many staging systems have been developed to guide the prognosis and treatment of patients with hepatocellular carcinoma (HCC) or intrahepatic cholangiocarcinoma (ICC), including the Barcelona Clinic Liver Cancer, ${ }^{7}$ Hong Kong Liver Cancer, ${ }^{8}$ American Joint Committee on Cancer (AJCC) $7^{\text {th }}$ edition, ${ }^{9}$ Nathan ${ }^{10}$ and Liver Cancer Study Group of Japan staging systems, ${ }^{11}$ but 
none for CHC patients. Previously, we established a novel risk prediction model that could be applied to facilitate the diagnosis of CHC patients in contrast to HCC or ICC patients. ${ }^{12}$ Further, we observed that surgical resection could provide the same outcome between elderly and younger patients. ${ }^{13}$ Though CHCs share similar clinical and pathological characteristics (mean age, positive viral hepatitis and solitary tumors) with HCC patients, the features of $\mathrm{CHC}$ are genetically closer to that of ICC. ${ }^{5,14}$ Considering the vastly different mechanisms of carcinogenesis and biological behavior, the current cancer classification for HCC or ICC may not be suitable for $\mathrm{CHC}$ patients. Thus, developing an accurate recurrence prediction model would make a contribution to treatment for CHC patients postoperatively.

In the present study, our aim was to establish a prognostic estimation of CHCs after resection (PECAR) score predicting recurrence on the basis of the clinicopathological data from Zhongshan Hospital and validate with an external cohort from Mengchao Hepatobiliary Hospital in China.

\section{Materials and methods}

\section{Patients}

The retrospective analysis obtained ethical approval and complied with the standards of the Declaration of Helsinki and current ethical guidelines. Informed consent was obtained from each patient before surgery for using their data for research. The $\mathrm{CHC}$ was histologically defined according to the WHO criteria, ${ }^{15}$ and two independent pathologists reassessed all these samples. The standard technique was adopted for hepatic resection. ${ }^{16}$ The probability of hilar lymph node metastasis was evaluated with preoperative contrast-enhanced computed tomography (CT) and (or) magnetic resonance imaging (MRI). The dissected lymphoid metastasis was routinely confirmed by pathology. Patients with no previous antitumor therapy, confirmed with $\mathrm{CHC}$ pathologically, no other malignancies and Child-Pugh class A or B were included in this study. The exclusion criteria were as follows: ICC or HCC proved by histopathology, tumors of uncertain origin, metastatic liver tumors, perioperative mortality and distant or intrahepatic metastasis. In the derivation cohort, $208 \mathrm{CHC}$ patients who underwent liver resection, between April 1999 and December 2014, at Zhongshan Hospital of Fudan University were enrolled. Further, we included an external validation cohort with 101 patients, from September 2003 to January 2016, at
Mengchao Hepatobiliary Hospital of Fujian Medical University. The study complied with the standards of the Declaration of Helsinki. The institutional review board of Zhongshan Hospital approved this study. All patients gave written informed consent.

\section{Laboratory test and data collection}

The serum chemistries, blood cell count and tumor biomarkers (serum alpha-fetoprotein [AFP], carcinoembryonic antigen [CEA], carbohydrate antigen 19-9 [CA19-9]) were measured in routine examination according to standard laboratory procedures. ${ }^{17}$ Hepatitis B surface antigen (HBsAg) and antibodies to hepatitis $\mathrm{C}$ virus $(\mathrm{HCV})$ were detected using standard test systems. To evaluate the potential risk predictors, all the data associated with demographic and pathological information were collected at the time of initial diagnosis.

\section{Follow-up and detection of recurrence}

All patients underwent follow-up every 3 months in the first year and every 6 months thereafter until death or dropout. Abdominal ultrasound, liver function tests, serum AFP, CEA and CA19-9 levels were analyzed every 3 months, and abdominal MRI or CT scanning was performed every 6 months. According to standard guidelines for $\mathrm{HCC}^{18}{ }^{18} \mathrm{ICC}^{19}$ or radiologic features of $\mathrm{CHC}$ described previously, ${ }^{20}$ recurrence was confirmed by contrast-enhanced imaging studies and tumor biomarkers.

Recurrent patients were managed with different therapeutic modalities, including radiofrequency ablation, repeated resection, transcatheter arterial chemoembolization (TACE) and supportive therapy. Overall survival (OS) was defined as the time period between the date of surgery and death and disease-free survival (DFS) was defined as the interval between the date of surgery and recurrence.

\section{Statistical analysis}

Continuous variables were reported as medians with IQR and categorical variables were as percentages. Pearson's $\chi^{2}$ test and Fisher's exact test were employed to compare categorical variables, whereas Wilcoxon test was used to evaluate continuous variables. Survival curves were computed with Kaplan-Meier methods and compared by using log-rank tests. Cox proportional hazards regression models were performed to determine univariate and multivariable HRs for predicting factors of $\mathrm{CHC}$ recurrence or survival. Predictors $(P<0.10$ in univariate analysis $)$ were selected in the multivariate analysis. The final multivariate model was 
performed using a backward stepwise procedure for variable selection with a liberal $P<0.05$ as the retention criteria. According to the final multivariable model coefficients, ${ }^{21}$ the novel prognostic score was developed, assigning ordinal scores ( 0 or 1$)$ to each of the selected factors. This simplified point scale could reflect the relative impact of risk covariables in the new model. And then, the relative value of each model component was summed to calculate the PECAR score. The PECAR score was tested and compared with AJCC $7^{\text {th }}$ TNM system in the validation cohort. We used the $\mathrm{C}$-index ${ }^{22}$ to assess model discrimination and absolute net reclassification index $(\mathrm{NRI})^{23,24}$ to evaluate the improvement of model performance for $\mathrm{CHC}$ recurrence. All statistical analyses were performed using SAS version 9.4 (SAS Institute Inc., Cary, NC, USA) and R software version 3.30 (R Foundation for Statistical Computing, Vienna, Austria). A $P$-value $<0.05$ was considered statistically significant.

\section{Results}

\section{Patient characteristics}

Table 1 presents the baseline characteristics of the derivation cohort $(n=208)$ and the validation cohort $(n=101)$. There were no differences in the baseline characteristics of the derivation and validation cohort. Patients were younger, male predominant and more likely to have a single tumor in both groups. Hepatitis B virus (HBV)-positive patients made up $64.9 \%$ of the derivation cohort and $69.3 \%$ of the validation cohort, respectively. Compared with the validation set, the derivation set tended to have a high proportion of adjuvant TACE after liver resection.

\section{Outcomes of $\mathrm{CHC}$ patients}

Among the entire cohort, the median OS was 18.1 months (range, 1.6-192.5 months). The 1-, 3- and 5-year OS probability was $76.2 \%, 41.2 \%$ and $28.3 \%$, respectively (Figure S1). The median RFS was 11.0 months (range, 1.0 to 192.5 ). The 1-, 3- and 5-year RFS probability was $48.0 \%, 37.8 \%$ and $28.0 \%$, respectively. Till to the date of last follow-up, 171 (55.3\%) patients died and 149 (48.2\%) patients encountered tumor relapse. There was no significant difference in the $\mathrm{CHC}$ recurrence between two groups (Figure 1).

\section{Predictor selection and construction of the PECAR score}

By using univariate analysis, male sex, elevated $\gamma$-glutamyl transferase (GGT), bleeding volume, tumor diameter and presence of macrovascular invasion, confirmed with hilar lymphoid metastasis and adjuvant TACE, at the first month after surgery were associated with subsequent $\mathrm{CHC}$ recurrence in the derivation cohort (Table 2). On multivariate analysis, the predictors that independently correlated with $\mathrm{CHC}$ recurrence included (1) sex, (2) GGT (at the following cutoffs: 0-39.9 and 240), (3) macrovascular invasion, (4) hilar lymphoid metastasis and (5) adjuvant TACE. The multivariate model coefficients for these 5 significant variables were transformed into relative points, and then summed to calculate the PECAR score as listed in Table 3. By adding total points for these 5 variables, the individualized PECAR score is calculated (Table 3).

\section{Predictive performance and discrimination}

The PECAR score ranged from 0 to 4 , with the most common score being 1 and 2 (Table 4). A female patient with $\mathrm{CHC}$ after liver resection, no macrovascular invasion, no hilar lymphoid metastasis and the GGT level lower than $40 \mathrm{U} / \mathrm{L}$ would have a PECAR score of 0 , predicting 1- and 5-year recurrence risk of only $11.1 \%$ and $22.2 \%$, respectively. Predicted risk of 1 - and 5-year $\mathrm{CHC}$ recurrence rose with each point score (Figure 2), such as a patient with a PECAR score of 4 or more, had a predicted 1- and 5-year recurrence risk of $72.7 \%$ and $81.8 \%$, respectively. The C-index of PECAR score was 0.651 (95\% CI, 0.593-0.710) in the derivation cohort for predicting CHC recurrence compared with AJCC $7^{\text {th }} \mathrm{TNM}$ staging system 0.520 (95\% CI, 0.465-0.575). By using the NRI, we found that the PECAR score improved prediction of $\mathrm{CHC}$ recurrence after liver resection compared with AJCC $7^{\text {th }}$ TNM staging system at 1 year $(0.194$, $P=0.002)$ and 5 years $(0.407, P<001)$ after liver resection.

\section{Validation of the PECAR score}

To validate whether the PECAR score would be applicable to other datasets, we conducted an external validation study with $101 \mathrm{CHC}$ patients in the validation group. In the validation cohort, median OS and RFS were 17.0 (1.8, $152.0)$ and $10.8(1.0,152.0)$ months, respectively. The $\mathrm{C}$-index of the PECAR score for predicting postoperative recurrence was 0.610 (95\% CI, 0.524-0.697), while the C-index of AJCC $7^{\text {th }}$ TNM staging system was 0.598 (95\% CI, 0.519-0.678). Similarly, the PECAR score improved the performance of recurrence prediction on 
Table I Demographic, clinical and tumor characteristics of patients with combined hepatocellular cholangiocarcinoma

\begin{tabular}{|c|c|c|c|}
\hline $\begin{array}{l}\text { Patient } \\
\text { demographics }\end{array}$ & $\begin{array}{c}\text { Derivation } \\
\text { cohort } \\
(n=208)\end{array}$ & $\begin{array}{c}\text { Validation } \\
\text { cohort } \\
(n=101)\end{array}$ & $P$-value \\
\hline \multicolumn{4}{|l|}{ Age, years } \\
\hline$<60$ & I 38 (66.35) & $65(64.36)$ & 0.83 \\
\hline$\geq 60$ & $70(33.65)$ & $36(35.64)$ & \\
\hline Sex (female), n (\%) & $64(30.77)$ & $27(26.73)$ & 0.55 \\
\hline HBV (positive), n (\%) & $135(64.9)$ & $70(69.31)$ & 0.52 \\
\hline HCV (positive), n (\%) & $205(98.56)$ & 99 (98.02) & 0.66 \\
\hline Liver cirrhosis, yes (\%) & $129(62.02)$ & $60(59.4 I)$ & 0.75 \\
\hline \multicolumn{4}{|l|}{ AFP, $\mathrm{ng} / \mathrm{mL}$} \\
\hline$<20$ & $104(50)$ & $55(54.46)$ & 0.45 \\
\hline $20-199$ & $48(23.08)$ & $17(16.83)$ & \\
\hline$\geq 200$ & $56(26.92)$ & $29(28.7 I)$ & \\
\hline \multicolumn{4}{|l|}{ CEA, $\mu g / m L$} \\
\hline$<5$ & I 57 (75.48) & $76(75.25)$ & 0.99 \\
\hline$\geq 5$ & $51(24.52)$ & $25(24.75)$ & \\
\hline \multicolumn{4}{|l|}{ CAI9-9, U/mL } \\
\hline$<37$ & $112(53.85)$ & $56(55.45)$ & 0.46 \\
\hline 37-399 & $65(31.25)$ & $35(34.65)$ & \\
\hline$\geq 400$ & $31(14.9)$ & $10(9.9)$ & \\
\hline \multicolumn{4}{|l|}{ Albumin, $g / L$} \\
\hline$<35$ & $19(9.13)$ & $3(2.97)$ & 0.06 \\
\hline$\geq 35$ & I 89 (90.87) & $98(97.03)$ & \\
\hline \multicolumn{4}{|l|}{ Bilirubin, $\mu \mathrm{mol} / \mathrm{L}$} \\
\hline$<17$ & 16I (77.4) & 74 (73.27) & 0.51 \\
\hline$\geq 17$ & $47(22.6)$ & $27(26.73)$ & \\
\hline \multicolumn{4}{|l|}{ ALT, IU/L } \\
\hline$<40$ & $124(59.62 \%)$ & 78 (77.23\%) & 0.003 \\
\hline$\geq 40$ & $84(40.38 \%)$ & 23 (22.77\%) & \\
\hline \multicolumn{4}{|l|}{ GGT, U/L } \\
\hline$<40$ & $48(23.08)$ & $34(33.66)$ & 0.07 \\
\hline$\geq 40$ & $160(76.92)$ & $67(66.34)$ & \\
\hline \multicolumn{4}{|l|}{ ALP, U/L } \\
\hline$<200$ & $199(95.67)$ & $99(98.02)$ & 0.51 \\
\hline$\geq 200$ & $9(4.33)$ & $2(1.98)$ & \\
\hline Prothrombin time, s & $12.3(11.4,13.2)$ & $12.1(1 \mid .4,13.0)$ & 0.49 \\
\hline \multicolumn{4}{|l|}{ PLT, $10^{3} / \mu \mathrm{L}$} \\
\hline$<100$ & $46(22.12)$ & $12(11.88)$ & 0.045 \\
\hline$\geq 100$ & $162(77.88)$ & $89(88.12)$ & \\
\hline \multicolumn{4}{|l|}{ Tumor number, n (\%) } \\
\hline 1 & $160(76.9)$ & $81(78.0)$ & 0.76 \\
\hline 2 & $24(11.5)$ & $9(8.9)$ & \\
\hline
\end{tabular}

(Continued)
Table I (Continued).

\begin{tabular}{|c|c|c|c|}
\hline $\begin{array}{l}\text { Patient } \\
\text { demographics }\end{array}$ & $\begin{array}{l}\text { Derivation } \\
\text { cohort } \\
(n=208)\end{array}$ & $\begin{array}{l}\text { Validation } \\
\text { cohort } \\
(n=101)\end{array}$ & $P$-value \\
\hline$\geq 3$ & $24(11.5)$ & II (I0.9) & \\
\hline Tumor diameter, cm & $5.3(3.5,8.0)$ & $5.0(3.0,7.5)$ & 0.19 \\
\hline $\begin{array}{l}\text { Microvascular inva- } \\
\text { sion (yes), n (\%) }\end{array}$ & $46(22.12)$ & $30(29.7)$ & 0.19 \\
\hline $\begin{array}{l}\text { Macrovascular inva- } \\
\text { sion (yes), n (\%) }\end{array}$ & $15(7.21)$ & $10(9.90)$ & 0.55 \\
\hline $\begin{array}{l}\text { Hilar lymphoid } \\
\text { metastasis (negative), } \\
\text { n (\%) }\end{array}$ & $22(10.58)$ & II (0.89) & 0.99 \\
\hline $\begin{array}{l}\text { Tumor differentiation } \\
\text { Well } \\
\text { Moderate } \\
\text { Poor }\end{array}$ & $\begin{array}{l}24(11.54) \\
134(64.42) \\
50(24.04)\end{array}$ & $\begin{array}{l}9(8.91) \\
61(60.40) \\
31(30.69)\end{array}$ & 0.41 \\
\hline $\begin{array}{c}\text { Bleeding } \\
\text { volume, } \mathrm{mL}\end{array}$ & $200(100,500)$ & $200(100,500)$ & 0.11 \\
\hline Occlusion, mins & $8.0(0.0,15.0)$ & $8.0(0.0,15.0)$ & 0.41 \\
\hline $\begin{array}{l}\text { Adjuvant TACE } \\
\text { No } \\
\text { Yes }\end{array}$ & $\begin{array}{l}155(74.52) \\
53(25.48)\end{array}$ & $\begin{array}{l}86(85.15) \\
15(14.85)\end{array}$ & 0.049 \\
\hline
\end{tabular}

Notes: Values are presented as $n(\%)$ or median (QI, Q3).

Abbreviations: HBV, hepatitis B virus; HCV, hepatitis C virus; AFP, $\alpha$-fetoprotein; CEA, carcinoembryonic antigen; CAI9-9, carbohydrate 19-9; ALT, alanine aminotransferase; GGT, $\gamma$-glutamyl transpeptidase; ALP, alkaline phosphatase; PLT, platelet; TACE, transcatheter arterial chemoembolization.

CHC compared with AJCC $7^{\text {th }}$ TNM staging system in the validation cohort ( 1 year: $0.185, P<0.001 ; 5$ years: 0.425 , $P=0.03)$.

\section{Discussion}

$\mathrm{CHC}$ is an uncommon liver tumor with distinctive biological behavior and clinicopathological features. Due to its rarity, the clinical information is limited, especially for the patients' survival and recurrence. In the present study, we developed and externally validated a novel prognostic score for postoperative CHC patients. This final model was based on 5 independent predictors with $\mathrm{C}$-index of 0.651 (95\%CI, 0.593-0.710) and presented better performance in recurrence prediction than AJCC $7^{\text {th }}$ TNM staging system.

Growing evidences document $\mathrm{CHC}$ as an aggressive cancer with dismal prognosis, and the tumor recurred frequently at the liver. ${ }^{16,25,26}$ Previously, we demonstrated 


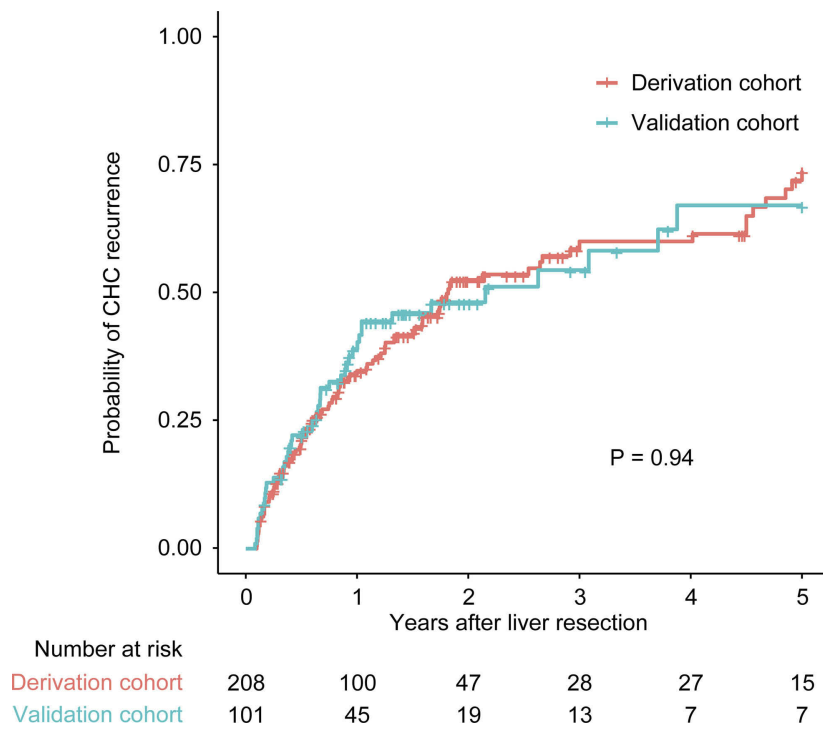

Figure I Kaplan-Meier probability of combined hepatocellular cholangiocarcinoma $(\mathrm{CHC})$ recurrence within 5 years in the derivation and validation cohorts. The number at risk refers to the numbers of patients who have not relapsed at the corresponding time point.

that $\mathrm{CHC}$ had a median prognosis between $\mathrm{HCC}$ and ICC. ${ }^{4}$ Further, we observed that the CHC patients had a short OS (18.1 months) and DFS (11.0 months) similar to previous studies. ${ }^{5,27} \mathrm{CHC}$ was thought to be derived from hepatic progenitor cells with the biopotential to differentiate into both hepatocytes and cholangiocytes. ${ }^{6,28}$ Aoki et $\mathrm{al}^{20}$ reported that the prognosis of $\mathrm{CHC}$ might be like that of mass-forming ICC, though the clinical characteristics of $\mathrm{CHC}$ are similar to those of HCC. However, several studies indicated that there was no relationship between poor outcome and the predominance of ICC cells (or HCC cells). ${ }^{29,30}$ Further studies are needed to investigate the intrinsic mechanism of $\mathrm{CHC}$.

Definitive evaluation of recurrent predictors in resected $\mathrm{CHC}$ patients has not been well established. Previous studies found that tumor number, vascular invasion, radical resection, lymphoid metastasis and tumor stage were prognostic factors for $\mathrm{CHC}$ patients. ${ }^{1,3,4,25} \mathrm{Kim}$ et al also reported that CA19-9 was a risk predictor for $\mathrm{CHC}$ patients. ${ }^{1}$ In our present study, five risk factors (sex, GGT, macrovascular invasion, lymphoid metastasis and adjuvant TACE) were identified as independent factors associated with recurrence. Interestingly, sex was selected as an independent risk factor related to $\mathrm{CHC}$ recurrence in our model, unlike tumor markers (serum AFP, CA19-9 or CEA). Previous studies indicated that $\mathrm{CHC}$ was more prevalent in male population, particularly in the endemic area of chronic hepatitis. ${ }^{25,31,32}$ The causative association between sex and recurrence of $\mathrm{CHC}$ patients is required to
Table 2 Univariate analysis of $\mathrm{CHC}$ recurrence in the derivation cohort $(n=208)$

\begin{tabular}{|c|c|c|}
\hline & $\begin{array}{l}\text { Univariable HR } \\
(95 \% \mathrm{Cl})\end{array}$ & $P$-value \\
\hline Sex (male vs female) & $1.884(1.192,2.979)$ & 0.007 \\
\hline Age ( $<60$ vs $\geq 60$ ), years & $1.297(0.86 \mathrm{I}, \mathrm{I} .958)$ & 0.22 \\
\hline HBsAg (positive vs negative) & $0.895(0.594,1.352)$ & 0.61 \\
\hline Liver cirrhosis (yes vs no) & I.188 (0.789, I.788) & 0.41 \\
\hline PLT ( $<100$ vs $\geq 100), 10^{3} / \mu \mathrm{L}$ & $1.051(0.664,1.663)$ & 0.83 \\
\hline Albumin (<35 vs $\geq 35$ ), g/L & $1.279(0.593,2.758)$ & 0.53 \\
\hline Bilirubin (<17 vs $\geq 17), \mu \mathrm{mol} / \mathrm{L}$ & $0.982(0.625,1.543)$ & 0.94 \\
\hline ALT (<40 vs $\geq 40)$, IU/L & $1.335(0.901,1.978)$ & 0.15 \\
\hline GGT ( $\geq 40$ vs $<40), U / L$ & $2.236(1.307-3.824)$ & 0.003 \\
\hline $\operatorname{ALP}(<200$ vs $\geq 200), U / L$ & $\mathrm{I} .364(0.554,3.36 \mathrm{I})$ & 0.54 \\
\hline AFP, ng/mL & & \\
\hline $20-199$ vs $<20$ & $1.219(0.754,0.674)$ & 0.42 \\
\hline$\geq 200$ vs $<20$ & $1.079(0.754,0.674)$ & 0.75 \\
\hline CEA ( $<5$ vs $\geq 5), \mu g / m L$ & $0.989(0.629,1.556)$ & 0.96 \\
\hline CAI9-9, U/mL & & \\
\hline $37-399$ vs $<37$ & $1.054(0.677,1.312)$ & 0.81 \\
\hline$\geq 400$ vs $<37$ & $1.406(0.745,1.847)$ & 0.22 \\
\hline Tumor number, $\mathrm{n}$ & $1.054(0.921,1.207)$ & 0.45 \\
\hline Tumor diameter, $\mathrm{cm}$ & $1.057(1.006-1.111)$ & 0.03 \\
\hline $\begin{array}{l}\text { Microvascular invasion (positive } \\
\text { vs negative) }\end{array}$ & $0.987(0.599,1.627)$ & 0.96 \\
\hline $\begin{array}{l}\text { Macrovascular invasion (positive } \\
\text { vs negative) }\end{array}$ & $3.383(1.746-6.554)$ & $<0.001$ \\
\hline $\begin{array}{l}\text { Hilar lymphoid metastasis (yes vs } \\
\text { no) }\end{array}$ & $2.306(1.276-4.168)$ & 0.006 \\
\hline Tumor differentiation & & \\
\hline Moderate vs poor & I.25I $(0.658, I .78 I)$ & 0.53 \\
\hline Well vs poor & $0.994(0.894,1.542)$ & 0.99 \\
\hline Bleeding volume, $\mathrm{mL}$ & $1.000(1.000,1.001)$ & 0.009 \\
\hline Adjuvant TACE (yes vs no) & 1.721 (1.126-2.632) & 0.01 \\
\hline
\end{tabular}

Abbreviations: AFP, $\alpha$-fetoprotein; CEA, carcinoembryonic antigen; CA 19-9, carbohydrate 19-9; ALT, alanine aminotransferase; GGT, $\gamma$-glutamyl transpeptidase; ALP, alkaline phosphatase; PLT, platelet; $\mathrm{HBsAg}$, hepatitis B surface antigen; $\mathrm{CHC}$, combined hepatocellular carcinoma; TACE, transcatheter arterial chemoembolization.

be confirmed in future studies. Consistent with previous studies, ${ }^{5,33}$ GGT, a risk factor of liver cancer, was found to be associated with RFS in our study. Macroscopic vascular invasion, the essential causes of intrahepatic recurrence 
Table 3 Multivariate analysis of risk factors of $\mathrm{CHC}$ recurrence and creation of the PECAR score $(n=208)$

\begin{tabular}{|c|c|c|c|c|}
\hline & Multivariable HR (95\% Cl) & $P$-value & $\beta$ coefficient & PECAR points \\
\hline \multicolumn{5}{|l|}{ Sex } \\
\hline Female & I (reference) & NA & NA & 0 \\
\hline Male & $1.815(1.315,2.903)$ & 0.01 & 0.596 & $\mathrm{I}$ \\
\hline \multicolumn{5}{|l|}{ GGT, U/mL } \\
\hline$<40$ & I (reference) & NA & NA & 0 \\
\hline$\geq 40$ & $1.932(1.114,3.351)$ & 0.02 & 0.659 & I \\
\hline \multicolumn{5}{|c|}{ Macrovascular invasion } \\
\hline Negative & I (reference) & NA & NA & 0 \\
\hline Positive & $2.966(1.484,5.93)$ & 0.002 & 1.087 & I \\
\hline \multicolumn{5}{|c|}{ Lymphoid metastasis } \\
\hline No & I (reference) & NA & NA & 0 \\
\hline Yes & $2.443(1.34 I, 4.45)$ & 0.004 & 0.893 & I \\
\hline
\end{tabular}

Abbreviations: GGT, $\gamma$-glutamyl transpeptidase; $\mathrm{CHC}$, combined hepatocellular cholangiocarcinoma; NA, not applicable; PECAR, prognostic estimation of CHCs after resection.

Table 4 The percentage of PECAR score in the derivation and the validation cohort

\begin{tabular}{|l|l|l|l|}
\hline Score & Derivation cohort & Validation cohort & P-value \\
\hline 0 & $18(8.6)$ & $12(11.9)$ & 0.74 \\
1 & $45(21.6)$ & $25(24.8)$ & \\
2 & $97(46.6)$ & $43(42.6)$ & \\
3 & $37(17.8)$ & $18(17.8)$ & \\
4 & $11(5.3)$ & $3(3.0)$ & \\
\hline
\end{tabular}

Notes: Values are presented as $\mathrm{n}(\%)$.

Abbreviations: CHC, combined hepatocellular cholangiocarcinoma; PECAR, prognostic estimation of $\mathrm{CHCs}$ after resection.

and long-term survival, was also identified as an independent predictor of $\mathrm{CHC}$ recurrence in accordance with previous studies in $\mathrm{HCC}^{34,35}$ and ICC. ${ }^{36}$ In liver cancer, Wang et $\mathrm{al}^{37}$ revealed that the prognosis of patients with lymph node metastasis or direct invasion and local metastasis was significantly poor. Also, lymphoid metastasis was found to be an independent predictor of recurrence with a HR of 2.443 (95\% CI, 1.341-4.450) in the present study. Extrahepatic recurrence was reported mainly in lymph nodes of $\mathrm{CHC}$ patients, ${ }^{5}$ suggesting that regional nodal groups need to be resected during operation.

Different from HCC, the pathological pattern of $\mathrm{CHC}$ is less vascular and much more fibrotic tissues, resulting in poor uptake of chemotherapeutic agents. Retrospective studies have shown that TACE could improve survival outcomes in recurrent or unresectable CHC patients, ${ }^{38,39}$ especially for hypervascular lesions. However, in patients with peripherally enhancing lesions, the prognosis was worse than HCC or globally enhancing CHC patients. ${ }^{39}$ In our study, we found that adjuvant TACE did not have a preventive effect, but may
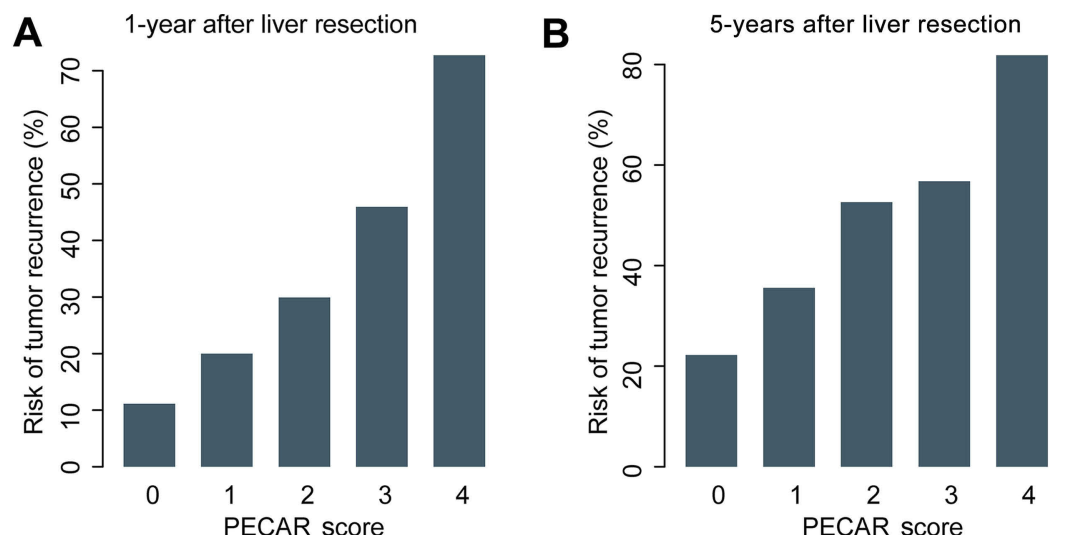

C $\begin{gathered}\text { Predicted } \mathrm{CHC} \text { recurrence risk of PECAR } \\
\text { score at 1- and 5-years }\end{gathered}$
\begin{tabular}{ccc} 
Score & 1-year & 5 -years \\
\hline 0 & $11.1 \%$ & $22.2 \%$ \\
1 & $20.0 \%$ & $35.6 \%$ \\
2 & $29.9 \%$ & $52.6 \%$ \\
3 & $45.9 \%$ & $56.8 \%$ \\
4 & $72.7 \%$ & $81.8 \%$ \\
\hline
\end{tabular}

Figure 2 Risk estimation of tumor recurrence in different PECAR scores after liver resection.

Notes: (A) The risk of tumor recurrence at I-year. (B) The risk of tumor recurrence at 5-years. (C) Predicted CHC recurrence risk of PECAR score at I-year and 5-years. Abbreviations: $\mathrm{CHC}$, combined hepatocellular cholangiocarcinoma; PECAR, prognostic estimation of $\mathrm{CHCs}$ after resection. 
increase the risk of recurrence for resected $\mathrm{CHC}$ patients. The explanations may be as follows: first, patients benefiting from adjuvant TACE were mainly combined with an intermediate or high risk of recurrence, ${ }^{17}$ second, $\mathrm{CHC}$ is an uncommon tumor with significant heterogeneity and aggressive biological behavior, patients with residual tumor may not respond to postoperative TACE; third, in our study, nearly $60 \%$ of patients combined with chronic HBV infection, the injury derived from TACE may promote local recurrence of CHC. Further investigation will be needed to verify the clinical efficacy of adjuvant TACE in CHC patients after hepatectomy.

How does our constructed model potentially influence clinical practice for CHC patients? First, it could help clinicians predict the likelihood of recurrence in postoperative $\mathrm{CHC}$ patients. In the present study, the probability of recurrence in patients with score $0,1,2,3$ and 4 at 1 year was $11.1 \%, 20.0 \%, 29.9 \%, 45.9 \%$ and $72.7 \%$, respectively. Second, the PECAR score will help us to determine whether $\mathrm{CHC}$ surveillance after resection is warranted. Previous studies indicated that the prognosis of CHC may be worse than HCC or ICC patients, implying that the surveillance should be modified and fitted for $\mathrm{CHC}$ patients in clinical practice. Currently, the clinical efficacy of therapeutic strategies on $\mathrm{CHC}$ after surgery remains unclear, and our PECAR score may facilitate the improvement of postoperative management of patients with intermediate or high risk of recurrence in the future.

This study is not devoid of limitations. First, the study is based on a retrospective cohort in China, with over $60 \%$ of patients having HBV infection; prospective studies in different populations are required to further validate our model. Second, our predictive model is used for postoperative decision-making. Third, genomic analyses were not performed for resected tumor specimens. Genomic classification has been shown to provide unique prognostic information, except for clinical parameters, and may help to identify patients at higher risk for subsequent metastatic tumor formation.

\section{Conclusions}

In conclusion, we constructed and validated a novel PECAR score predicting the recurrence of HBV-related CHC patients in stage I/II. The PECAR score may help predict the per-patient recurrence risk and facilitate clinicians manage patients with stage I/II CHC patients, and further studies are also needed to improve clinical strategy.

\section{Abbreviations list}

CHC, combined hepatocellular cholangiocarcinoma; HCC, hepatocellular carcinoma; ICC, intrahepatic cholangiocarcinoma; OS, overall survival; DFS, diseasefree survival; BCLC, Barcelona Clinic Liver Cancer; HKLC, Hong Kong Liver Cancer; AJCC, American Joint Committee on Cancer; LCSGJ, Liver Cancer Study Group of Japan; HBV, hepatitis B virus; MRI, magnetic resonance imaging; CT, computed tomography; AFP, alpha-fetoprotein; CEA, carcinoembryonic antigen; CA19-9, carbohydrate antigen 19-9; PEI, percutaneous ethanol injection; C-index, concordance index; VI, vascular invasion.

\section{Acknowledgments}

We all express our sincere thanks to Zun-Song $\mathrm{Hu}$ for statistical analysis. We would like to thank the participants and staff of the Zhongshan Hospital for their valuable contributions; the staff included Zhen-Bin Ding, YuanFei Peng, Zhi Dai, and Pei-Yun Zhou. This work was supported by grants from the National Natural Science Foundation of China (81472674, 81773067, 81800790 and 81502486), the National Key Sci-Tech Project (2012ZX10002011-002), the Shanghai Committee of Science and Technology, China (No. 16JC1404000), the Shu Guang Project of Shanghai Municipal Education Commission and Shanghai Education Development Foundation (13SG04), and the China Postdoctoral Science Foundation Funded Project (2018M640343).

\section{Disclosure}

The authors declared no conflicts of interest in this work.

\section{References}

1. Kim KH, Lee SG, Park EH, et al. Surgical treatments and prognoses of patients with combined hepatocellular carcinoma and cholangiocarcinoma. Ann Surg Oncol. 2009;16(3):623-629. doi:10.1245/s10434-008-0278-3

2. Allen RA, Lisa JR. Combined liver cell and bile duct carcinoma. $A m$ J Pathol. 1949;25(4):647-655.

3. Jarnagin WR, Weber S, Tickoo SK, et al. Combined hepatocellular and cholangiocarcinoma: demographic, clinical, and prognostic factors. Cancer. 2002;94(7):2040-2046.

4. Yin X, Zhang BH, Qiu SJ, et al. Combined hepatocellular carcinoma and cholangiocarcinoma: clinical features, treatment modalities, and prognosis. Ann Surg Oncol. 2012;19(9):2869-2876. doi:10.1245/ s10434-012-2328-0

5. Lee SD, Park SJ, Han SS, et al. Clinicopathological features and prognosis of combined hepatocellular carcinoma and cholangiocarcinoma after surgery. Hepatobiliary Pancreatic Dis Int. 2014;13 (6):594-601. doi:10.1016/S1499-3872(14)60275-7 
6. Brunt E, Aishima S, Clavien PA, et al. cHCC-CCA: consensus terminology for primary liver carcinomas with both hepatocytic and cholangiocytic differentation. Hepatology (Baltimore, Md). 2018;68:113-126. doi:10.1002/hep.29789

7. Llovet JM, Bru C, Bruix J. Prognosis of hepatocellular carcinoma: the BCLC staging classification. Semin Liver Dis. 1999;19 (3):329-338. doi:10.1055/s-2007-1007122

8. Yau T, Tang VY, Yao TJ, Fan ST, Lo CM, Poon RT. Development of Hong Kong liver cancer staging system with treatment stratification for patients with hepatocellular carcinoma. Gastroenterology. 2014;146(7):1691-1700.e1693. doi:10.1053/j.gastro.2014.02.032

9. Farges O, Fuks D, Le Treut YP, et al. AJCC 7th edition of TNM staging accurately discriminates outcomes of patients with resectable intrahepatic cholangiocarcinoma: by the AFC-IHCC-2009 study group. Cancer. 2011;117(10):2170-2177. doi:10.1002/cncr.25712

10. Nathan H, Aloia TA, Vauthey JN, et al. A proposed staging system for intrahepatic cholangiocarcinoma. Ann Surg Oncol. 2009;16 (1):14-22. doi:10.1245/s10434-008-0180-z

11. Yamasaki S. Intrahepatic cholangiocarcinoma: macroscopic type and stage classification. J Hepatobiliary Pancreat Surg. 2003;10 (4):288-291. doi:10.1007/s00534-002-0732-8

12. Tian MX, He WJ, Liu WR, et al. A novel risk prediction model for patients with combined hepatocellular-cholangiocarcinoma. J Cancer. 2018;9(6):1025-1032. doi:10.7150/jca.23229

13. Tao CY, Liu WR, Jin L, et al. Surgical treatment of combined hepatocellular-cholangiocarcinoma is as effective in elderly patients as it is in younger patients: a propensity score matching analysis. $J$ Cancer. 2018;9(6):1106-1112. doi:10.7150/jca.23921

14. Cazals-Hatem D, Rebouissou S, Bioulac-Sage P, et al. Clinical and molecular analysis of combined hepatocellular-cholangiocarcinomas. J Hepatol. 2004;41(2):292-298. doi:10.1016/j.jhep.2004.04.030

15. Hurlimann J, Gardiol D. Immunohistochemistry in the differential diagnosis of liver carcinomas. Am J Surg Pathol. 1991;15(3):280-288.

16. Liu CL, Fan ST, Lo CM, et al. Hepatic resection for combined hepatocellular and cholangiocarcinoma. Arch Surg (Chicago, Ill: 1960). 2003;138(1):86-90.

17. Wang Z, Ren Z, Chen Y, et al. Adjuvant transarterial chemoembolization for HBV-related hepatocellular carcinoma after resection: a randomized controlled study. Clin Cancer Res. 2018;24 (9):2074-2081. doi:10.1158/1078-0432.CCR-17-2899

18. European Association for the Study of the Liver. Electronic address eee, European association for the study of the L. EASL clinical practice guidelines: management of hepatocellular carcinoma. J Hepatol. 2018;69(1):182-236. doi:10.1016/j.jhep.2018.03.019

19. Bridgewater J, Galle PR, Khan SA, et al. Guidelines for the diagnosis and management of intrahepatic cholangiocarcinoma. $J$ Hepatol. 2014;60(6):1268-1289. doi:10.1016/j.jhep.2014.01.021

20. Aoki K, Takayasu K, Kawano T, et al. Combined hepatocellular carcinoma and cholangiocarcinoma: clinical features and computed tomographic findings. Hepatology (Baltimore, Md). 1993;18(5):1090-1095.

21. Sullivan LM, Massaro JM, D“Agostino RB Sr. Presentation of multivariate data for clinical use: the framingham study risk score functions. Stat Med. 2004;23(10):1631-1660. doi:10.1002/sim.1742

22. Brentnall AR, Cuzick J. Use of the concordance index for predictors of censored survival data. Stat Methods Med Res. 2018;27 (8):2359-2373. doi:10.1177/0962280216680245

23. Pencina MJ, Steyerberg EW, D`Agostino RB Sr. Net reclassification index at event rate: properties and relationships. Stat Med. 2017;36 (28):4455-4467. doi:10.1002/sim.7041

24. Kerr KF, Wang Z, Janes H, McClelland RL, Psaty BM, Pepe MS. Net reclassification indices for evaluating risk prediction instruments: a critical review. Epidemiology. 2014;25(1):114-121. doi:10.1097/ EDE.0000000000000018
25. Koh KC, Lee H, Choi MS, et al. Clinicopathologic features and prognosis of combined hepatocellular cholangiocarcinoma. $\mathrm{Am}$ J Surg. 2005;189(1):120-125. doi:10.1016/j.amjsurg.2004.03.0 18

26. Yeh MM. Pathology of combined hepatocellular-cholangiocarcinoma. $J$ Gastroenterol Hepatol. 2010;25(9):1485-1492. doi:10.1111/j.14401746.2010.06430.x

27. Sapisochin G, Fidelman N, Roberts JP, Yao FY. Mixed hepatocellular cholangiocarcinoma and intrahepatic cholangiocarcinoma in patients undergoing transplantation for hepatocellular carcinoma. Liver Transplant. 2011;17(8):934-942. doi:10.1002/lt.22307

28. Cai X, Zhai J, Kaplan DE, et al. Background progenitor activation is associated with recurrence after hepatectomy of combined hepatocellular-cholangiocarcinoma. Hepatology (Baltimore, Md). 2012;56(5):1804-1816. doi:10.1002/hep.25874

29. Ariizumi S, Kotera Y, Katagiri S, Nakano M, Yamamoto M. Combined hepatocellular-cholangiocarcinoma had poor outcomes after hepatectomy regardless of Allen and Lisa class or the predominance of intrahepatic cholangiocarcinoma cells within the tumor. Ann Surg Oncol. 2012;19(5):1628-1636. doi:10.1245/ s10434-011-2150-0

30. Shin CI, Lee JM, Kim SH, et al. Recurrence patterns of combined hepatocellular-cholangiocarcinoma on enhanced computed tomography. J Comput Assist Tomogr. 2007;31(1):109-115. doi:10.1097/01.rct.0000235072.34808.9b

31. Akiba J, Nakashima O, Hattori S, et al. Clinicopathologic analysis of combined hepatocellular-cholangiocarcinoma according to the latest WHO classification. Am J Surg Pathol. 2013;37(4):496-505. doi:10.1097/PAS.0b013e31827332b0

32. Shibahara J, Hayashi A, Misumi K, et al. Clinicopathologic characteristics of hepatocellular carcinoma with reactive ductule-like components, a subset of liver cancer currently classified as combined hepatocellular-cholangiocarcinoma with stem-cell features, typical subtype. Am J Surg Pathol. 2016;40:608-616. doi:10.1097/ PAS.0000000000000579

33. Mok Y, Son DK, Yun YD, Jee SH, Samet JM. gamma-glutamyltransferase and cancer risk: the Korean cancer prevention study. Int J Cancer J Int Du Cancer. 2016;138(2):311-319. doi:10.1002/ijc.29659

34. Shim JH, Jun MJ, Han S, et al. Prognostic nomograms for prediction of recurrence and survival after curative liver resection for hepatocellular carcinoma. Ann Surg. 2015;261(5):939-946. doi:10.1097/ SLA.0000000000000747

35. Zhong JH, Ke Y, Gong WF, et al. Hepatic resection associated with good survival for selected patients with intermediate and advanced-stage hepatocellular carcinoma. Ann Surg. 2014;260 (2):329-340. doi:10.1097/SLA.0000000000000236

36. Hyder $\mathrm{O}$, Marques $\mathrm{H}$, Pulitano $\mathrm{C}$, et al. A nomogram to predict long-term survival after resection for intrahepatic cholangiocarcinoma: an Eastern and Western experience. JAMA Surg. 2014;149(5):432-438. doi:10.1001/jamasurg.2013.51 68

37. Wang Y, Li J, Xia Y, et al. Prognostic nomogram for intrahepatic cholangiocarcinoma after partial hepatectomy. $J$ Clin Oncol. 2013;31(9):1188-1195. doi:10.1200/JCO.2012.41.5984

38. Kim JH, Yoon HK, Ko GY, et al. Nonresectable combined hepatocellular carcinoma and cholangiocarcinoma: analysis of the response and prognostic factors after transcatheter arterial chemoembolization. Radiology. 2010;255(1):270-277. doi:10.1148/ radiol.09091076

39. Na SK, Choi GH, Lee HC, et al. The effectiveness of transarterial chemoembolization in recurrent hepatocellular-cholangiocarcinoma after resection. PLoS One. 2018;13(6):e0198138. doi:10.1371/journal. pone. 0198138 


\section{Supplementary material}

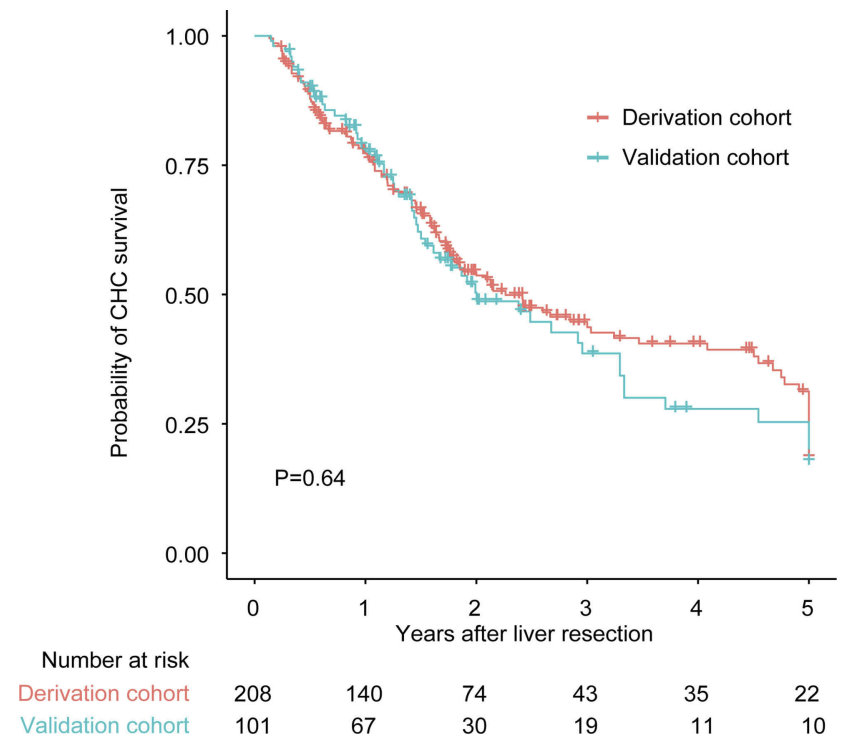

Figure SI Kaplan-Meier probability of Combined-hepatocellular carcinoma (CHC) overall survival within 5 years in the derivation and validation cohorts. The number at risk refers to the numbers of patients who have not died at the corresponding time point.

\section{Publish your work in this journal}

Cancer Management and Research is an international, peer-reviewed open access journal focusing on cancer research and the optimal use of preventative and integrated treatment interventions to achieve improved outcomes, enhanced survival and quality of life for the cancer patient.
The manuscript management system is completely online and includes a very quick and fair peer-review system, which is all easy to use. Visit http://www.dovepress.com/testimonials.php to read real quotes from published authors. 